Max-Planck-Institut für demografische Forschung

Max Planck Institute for Demographic Research

Konrad-Zuse-Strasse 1 - D-18057 Rostock - GERMANY

Tel +49 (0) 3812081 - 0; Fax +49 (0) 3812081 - 202;

http://www.demogr.mpg.de

MPIDR WORKING PAPER WP 2011-019

NOVEMBER 2011

Der Kinderwunsch im Kontext von

Partnerschaft und Partnerschafts-

qualität:

Eine Analyse übereinstimmender

Elternschaftsabsichten von Eltern und

kinderlosen Paaren

Julika Hillmann (Julika.Hillmann@lskn.niedersachsen.de)

Anne-Kristin Kuhnt (kuhnt@demogr.mpg.de)

This working paper has been approved for release by: Michaela Kreyenfeld (kreyenfeld@demogr.mpg.de), Acting Deputy Head of the Laboratory of Economic and Social Demography.

(C) Copyright is held by the authors.

Working papers of the Max Planck Institute for Demographic Research receive only limited review. Views or opinions expressed in working papers are attributable to the authors and do not necessarily reflect those of the Institute. 


\title{
Der Kinderwunsch im Kontext von Partnerschaft und Partnerschafts- qualität: Eine Analyse übereinstimmender Elternschaftsabsichten von Eltern und kinderlosen Paaren
}

Fertility intentions in the context of partnership and relationship quality: An analysis of dyadic fertility intentions of parents and childless couples

Julika Hillmann (Julika.Hillmann@ @skn.niedersachsen.de)

Anne-Kristin Kuhnt (kuhnt@demogr.mpg.de)

\section{Zusammenfassung}

Untersuchungen zur Kinderwunschthematik stellen bisher Individualanalysen in den Mittelpunkt, dabei wird der Partnerschaftskontext, in dem Kinder(wünsche) entstehen, vernachlässigt. Die vorliegende Arbeit untersucht anhand der Befragung von 2.793 Paaren im Rahmen der „Panel Analysis of Intimate Relationships and Family Dynamics“ (pairfam) die Übereinstimmung von Paaren hinsichtlich ihrer zeitnahen Elternschaftsabsichten unter Berücksichtigung der subjektiv wahrgenommenen Partnerschaftsqualität. Demnach hat eine hohe Partnerschaftsqualität einen positiven Einfluss auf den übereinstimmenden Kinderwunsch von Paaren. Betrachtet man Eltern und kinderlose Paare separat, wird deutlich, dass Elternschaftsabsichten von Paaren mit Kindern deutlich stärker von der Partnerschaftsqualität abhängen, als die von kinderlosen Paaren.

\section{Schlagworte}

Übereinstimmender Kinderwunsch von Paaren, Partnerschaftsqualität, Kinderlose Paare, Eltern

\begin{abstract}
Although many studies consider fertility intentions, research almost neglects dyadic family desires. Prior micro level studies have basically ignored the dyadic nature of fertility intentions. This study uses survey data from 2.793 couples of the German „Panel Analysis of Intimate Relationships and Family Dynamics“ (pairfam) to evaluate congruent fertility intentions of couples, and considers self-perceived relationship quality in particular. The results show that high relationship quality leads to a positive impact on congruent fertility intentions of couples. If parents and childless couples are considered separately, the results clarify that fertility intentions of parents depend more strongly on the relationship quality than those of childless couples.
\end{abstract}

\section{Keywords}

Congruent fertility intentions of couples, relationship quality, childless couples, parents 


\section{$1 \quad$ Einführung}

Obwohl die Analyse des Kinderwunsches zu den Kernthemen empirischer Familienforschung gehört, liegen kaum Befunde zum partnerschaftlichen Kinderwunsch vor. Untersuchungen erfolgen bisher für Deutschland und auch im internationalen Kontext fast ausschließlich auf Individualebene (z.B. Engelhardt 2004, Heiland et al. 2008, Hayford 2009), was nicht zuletzt einem Mangel an adäquaten Daten geschuldet ist (Rosina/Testa 2009: 489). Diese Betrachtungsweise vernachlässigt, dass generative Entscheidungen (in den meisten Fällen) im Kontext einer Paarbeziehung getroffen bzw. umgesetzt werden.

Wird der Kinderwunsch eines Einzelnen ohne den (vielleicht absolut gegenläufigen) Kinderwunsch des aktuellen Partners betrachtet, ist die Aussagekraft des Kinderwunsches als Messinstrument für zukünftige Elternschaftsabsichten eingeschränkt. Kann doch der individuelle Kinderwunsch gänzlich von dem im Partnerschaftskontext ausgehandelten abweichen. Vor diesem Hintergrund erscheint die Betrachtung von individuellen Kinderwünschen als keinesfalls ausreichend. Dennoch findet bisher kaum eine Differenzierung zwischen individuellen und partnerschaftlichen Kinderwünschen statt (Rosina/Testa 2009: 488; Peuckert 2008: 114).

An diesem Defizit setzt diese Arbeit an und rückt die Übereinstimmung von Paaren hinsichtlich ihrer zeitnahen Elternschaftsabsichten in den Mittelpunkt. Ergänzend dazu werden Attribute der Paarbeziehung, wie zum Beispiel die subjektiv wahrgenommene Beziehungsqualität, in diesem Beitrag berücksichtigt.

Bisherige Studien weisen einen Zusammenhang zwischen Aspekten der Partnerschaftsqualität und der Realisierung von Geburten nach (z.B. Rijken/Thomson 2010, Rijken 2009, Rijken/Liefbroer2009, Meyers 1997). Demnach steigt die Wahrscheinlichkeit der Geburt eines Kindes innerhalb einer 
Paarbeziehung, wenn die Interaktion zwischen den Partnern weder zu positiv noch zu negativ ist (Rijken/Liefbroer 2009: 27, Rijken 2009: 67). Dies lässt vermuten, dass bereits vor der Umsetzung von Fertilitätsintentionen ein Zusammenhang zwischen einzelnen Aspekten der Partnerschaftsqualität und der Ausbildung von Kinderwünschen besteht. Daher gehen wir in diesem Beitrag zuerst der Frage nach, ob und wie Aspekte der Partnerschaftsqualität auf einen übereinstimmenden Kinderwunsch von Paaren wirken. Weiterhin werden Kinderwünsche von Eltern, im Gegensatz zu Kinderwünschen von bislang Kinderlosen, zusätzlich durch bereits erworbene Erfahrungen mit den eigenen Kindern beeinflusst (siehe auch Heiland et al. 2008: 135, Jokela 2010: 915). Gleichzeitig scheint eine Wechselwirkung zwischen der Partnerschaftsqualität und dem Vorhandensein von Kindern zu bestehen, fällt doch die Partnerschaftsqualität von Eltern niedriger aus als die von Kinderlosen (Graf 2002: 111ff, Rijken 2009: 63). Daher interessiert uns als zweite zentrale Forschungsfrage, ob Unterschiede zwischen Eltern und kinderlosen Paaren hinsichtlich ihrer übereinstimmend geäußerten Absicht, in den nächsten zwei Jahren ein Kind zu bekommen, existieren.

Der vorliegende Beitrag präsentiert zunächst bisherige Forschungsergebnisse zum Einfluss der Partnerschaftsqualität auf den Kinderwunsch (Abschnitt 2). Anschließend werden theoretische Überlegungen zur Entstehung von Kinderwünschen dargestellt, die sich auf den Value of Children-Ansatz (Hoffman/Hoffman 1973) und die soziale Austauschtheorie (Thibaut/Kelley 1959) konzentrieren (Abschnitt 3). Auf dieser Grundlage werden die Hypothesen zum Einfluss der Partnerschaftsqualität auf den übereinstimmenden Kinderwunsch von Paaren formuliert (Abschnitt 3.1). In den darauf folgenden Abschnitten werden die Daten (Abschnitt 4), die Operationalisierung der erklärenden Variable Partnerschaftsqualität (Abschnitt 5) und die verwendeten Methoden (Abschnitt 6) 
sowie die Ergebnisse (Abschnitt 7) dargestellt. Der Artikel schließt mit einer Diskussion der Ergebnisse (Abschnitt 8).

\section{Forschungsstand zum Zusammenhang zwischen Partnerschaftsqualität und Kinderwunsch}

Die Qualität einer Beziehung scheint für Frauen und Männer entscheidend für die Geburt eines Kindes zu sein (Rijken/Liefbroer2009: 38), zur Wirkungsweise der Partnerschaftsqualität auf den Kinderwunsch existiert jedoch kaum Literatur. $\mathrm{Zu}$ den einzelnen Aspekten der Partnerschaftsqualität liegen vorrangig Forschungsergebnisse getrennt für Frauen und Männern vor, welche die Paarperspektive vernachlässigen. So zeigen Eckhard und Klein (2006, 2007), dass die Stabilität einer Paarbeziehung eher ein männerspezifischer Motivationsfaktor für eine Elternschaft ist. Männer empfinden eine stabile Beziehung stärker als Frauen als Voraussetzung für eine Elternschaft (ibd. 2006: 179). Eine bestehende Elternschaft hat demnach einen positiven Einfluss auf den Kinderwunsch von Männern, wenn diese keine Beziehungsprobleme wahrnehmen (ibd. 2007: 283ff.). Diese Befunde deuten an, dass die Partnerschaftsqualität besonders für die generative Entscheidung von Männern relevant ist aber weniger für die von Frauen. Gleichzeitig suggerieren diese geschlechtsspezifischen Unterschiede, dass in einer Partnerschaft nicht immer Konsens über den Kinderwunsch bestehen muss.

Weiterführend scheinen sich Elternschaftsabsichten von Kinderlosen und Eltern sowie deren Bestimmungsgründe voneinander zu unterscheiden (ibd. 2006: 181f, ibd. 2007: 285ff.). Dies könnte darauf zurückzuführen sein, dass sich bereits erworbene Elternschaftserfahrungen in der Wahrnehmung von Beweg- und Hinderungsgründen niederschlagen und sich damit auf den Wunsch nach weiteren Kindern auswirken (ibd. 2006: 79). Ein erstes Kind ist demnach mit weitaus 
stärkeren Konsequenzen für die Partnerschaft verbunden als Kinder höherer Ordnung (Bulatao 1981: 18, 23; Rijken 2009: 58). So bewerten Eltern die Gefahr von Partnerschaftskonflikten im Vergleich zu Kinderlosen als weniger gravierend (Klein/Eckhard 2008: 391f.). In einer Partnerschaft lebende Männer messen der Bedeutung von Konflikten in einer Beziehung als ein Hinderungsgrund einer (weiteren) Elternschaft mehr Gewicht als Frauen bei (ibd. 2008: 391f.). Ergebnisse von Lillard und Waite (1993: 656) verdeutlichen, dass ein hohes Trennungsrisiko die Elternschaftsabsichten verheirateter Paare deutlich reduzieren oder sogar ganz verhindern kann. Analysen auf Basis des Generations and Gender Survey (GGS) aus dem Jahr 2005 fügen sich in diese Ergebnisse ein und zeigen für Deutschland, dass Kinderwünsche von Männern und Frauen insbesondere dann divergieren, wenn es um die Geburt weiterer Kinder geht (Höhn et al. 2006: 38). Bei Eltern kann demnach eine starke Fokussierung auf den Partner dazu führen, dass weitere Kinder als Gefährdung der Beziehung bzw. Beziehungsqualität angesehen werden.

Eine aktuelle Studie über den Einfluss der Partnerschaftsqualität auf den Übergang zur Elternschaft von Rijken (2009) dokumentiert für die Niederlande, dass insbesondere Frauen mit einer mittelmäßigen Einschätzung der Qualität ihrer Partnerschaft ein erhöhtes Risiko für eine Erst- und Zweitgeburt aufweisen. Bei Männern hat die Einschätzung der Partnerschaftsqualität hingegen kaum Bedeutung für den Übergang zur Elternschaft. Allerdings ist auch ihr Risiko für die Geburt eines zweiten Kindes erhöht, wenn sie der Partnerschaft eine mittlere Qualität bescheinigen. Demnach bevorzugen beide Geschlechter die Geburt eines Kindes in einer Beziehung mit mittlerem Qualitätsniveau. Frauen, die ihren Beziehungen eine sehr hohe Qualität zusprechen, fürchten hingegen stärker als Männer negative Konsequenzen eines (weiteren) Kindes für ihre Partnerschaft. Frauen scheinen die Partnerschaftsqualität bereits bei der Familiengründung zu berücksichtigen, 
während diese für Männer erst bei der Frage nach einem zweiten Kind relevant wird.

Die existierenden Befunde zum Einfluss der Attribute einer Paarbeziehung auf generative Entscheidungsprozesse verdeutlichen somit, dass Männer und Frauen die Qualität ihrer Beziehung in unterschiedlicher Weise bei ihrer Fertilitätsentscheidung berücksichtigen. Gleichzeitig geht aus den Ergebnissen hervor, dass diesbezüglich auch Unterschiede zwischen Eltern und Kinderlosen bestehen. Um beide Kontroversen miteinander zu verbinden, werden im Folgenden zunächst Annahmen zur Wirkungsweise der Partnerschaftsqualität auf übereinstimmende Elternschaftsabsichten formuliert und anschließend zu den Unterschieden zwischen Eltern und kinderlosen Paaren.

\section{Theoretische Überlegungen}

Sowohl der Value of Children-Ansatz (VOC) (Hoffman/Hoffman 1973) als auch die soziale Austauschtheorie (Thibaut/Kelley 1959) können zur Beschreibung und Erklärung der Genese von Kinderwünschen herangezogen werden. Beiden Ansätzen ist ein handlungstheoretisches Modell zur Ausbildung von Kinderwünschen gemein. Die Modelle unterstellen jeweils rationale Akteure, die Handlungsoptionen anhand eines Kosten-Nutzen-Kalküls bewerten und sich an den dadurch zu erzielenden Gewinnen orientieren. Die Option Elternschaft, die damit verbundenen Kosten sowie die dazu möglichen Alternativen sind Komponenten dieser Modelle und werden einer Kosten-Nutzen-Abwägung unterzogen. Dadurch fließen sie in die generative Entscheidungsfindung ein. Die generative Entscheidung fällt dann positiv aus, wenn eine (weitere) Elternschaft subjektiv den größtmöglichen $\mathrm{zu}$ erzielenden Ertrag im Vergleich $\mathrm{zu}$ den verfügbaren Alternativen verspricht. 
Beide theoretischen Ansätze berücksichtigen motivationale Einflüsse auf die Ausbildung des Kinderwunsches. Dies unterscheidet sie von rein ökonomischen Modellen. Dabei heben beide Ansätze die Relevanz individueller bzw. paarspezifischer psychosozialer Besonderheiten im Rahmen der generativen Entscheidung hervor. Fertilitätsverhalten wird dabei auf der Basis von individuellen Präferenzen, Orientierungen und Einstellungen sowie den daraus abgeleiteten Verhaltens- und Entscheidungsregelmäßigkeiten erklärt (Huinink/Konietzka 2007: 152ff.). Zwar existieren empirische Belege dafür, dass der Zusammenhang zwischen Einstellung und Verhalten oft nur gering ist (Klaus/Suckow 2006: 103), dies ist jedoch nur für den Zusammenhang zwischen Fertilitätsabsichten und deren Umsetzung relevant, nicht aber für die Ausbildung der generativen Entscheidung.

Da der VOC-Ansatz die individuelle Kinderwunschgenese in den Mittelpunkt stellt, muss zur Analyse des Kinderwunsches im Paarkontext ein anderes Erklärungsmodell herangezogen werden. Das Paarinteraktionsmodell von Beckman (1977, 1982), das auf der sozialen Austauschtheorie beruht, ermöglicht es, sowohl Männer und Frauen einzeln als auch das Paar als Einheit, im generativen Entscheidungs- und Verhaltensprozess zu untersuchen. Das Modell unterscheidet von vornherein zwischen soziodemographischen und Persönlichkeitsfaktoren von Frauen und Männern, die zur Ausbildung unterschiedlicher Elternschaftsabsichten in Paarbeziehungen führen können. Weiterhin finden Paarinteraktion und Paarinterdependenz Berücksichtigung, wodurch es möglich ist, verschiedene Faktoren der Beziehung $\mathrm{zu}$ integrieren. Dazu zählen wechselseitige Beeinflussungen der Partner bei der Ausbildung ihrer Kinderwünsche, Merkmale der Paarbeziehung als Einflussfaktoren auf die Genese von Kinderwünschen, unterschiedliche Verhaltensintentionen der Partner im Prozess der Entscheidungsfindung und die Art und Weise, wie diese aufeinander abgestimmt 
werden (Borchardt/Stöbel-Richter 2004: 41).

\subsection{Hypothesen}

Durch Interaktion beeinflussen Partner ihre Motivation zur Elternschaft gegenseitig (Hill/Kopp 2004: 112; Lenz 1998: 31). Das Ausmaß dieser Interaktion wirkt ebenfalls auf die Partnerschaftsqualität. So können Merkmale der Paarbeziehung als Einfluss nehmende Faktoren einer generativen Entscheidung betrachtet werden. Wenn Männer und Frauen die Qualität ihrer Partnerschaft bei ihrer Entscheidung für oder gegen eine Elternschaft berücksichtigen, stellt sich die Frage, wie sich die Partnerschaftsqualität auf den Kinderwunsch von Männern und Frauen auswirkt.

Aus der sozialen Austauschtheorie geht hervor, dass die Partnerschaft an sich einen eigenen Wert für Individuen innehat, da sie eine Nutzen generierende Quelle der Bedürfnisbefriedigung darstellt (Schmidt/Moritz 2009: 33f.). Die Kosten, die mit der Geburt und dem Aufziehen eines (weiteren) Kindes verbunden sind, werden von beiden Partnern, wenn auch in unterschiedlicher Stärke, getragen (Nauck 2001: 408). Sie äußern sich bspw. durch psychische und emotionale Belastungen bei der Erziehung sowie individuelle (Unterbrechung oder Aufgabe der Erwerbstätigkeit) und partnerschaftliche Opportunitätskosten (weniger Zeit, die mit dem Partner verbracht werden kann).

Besonders unter dem Gesichtspunkt der gestiegenen Ansprüche an die aktuelle Partnerschaft können Kinder demnach als Konkurrenz zur Partnerschaft betrachtet werden (Peuckert 2008: 121). Werden Kinder mit einer Reduzierung des Nutzens, der aus einer Partnerschaft gewonnen werden kann, assoziiert, ist es unwahrscheinlich, dass Kinder gewünscht werden, wenn die Beziehungsqualität sehr hoch bewertet wird. 
Ergänzend dazu verdeutlicht der VOC-Ansatz, dass Kinder einen Wert für (potenzielle) Eltern haben, der individuelle Wohlfahrt generiert und den individuellen Nutzen steigern kann. In einer Partnerschaft, die keinen zufrieden stellenden Nutzen stiftet, die aufgrund mangelnder Handlungsalternativen (oder geltender Normen) aber auch nicht aufgelöst werden kann, könnte ein Kinderwunsch eine Steigerung des individuellen (Beziehungs-)Nutzens mit sich bringen. Diese beiden Argumentationsstränge führen zu der Annahme,

dass eine geringe Partnerschaftsqualität einen positiven Einfluss auf übereinstimmende Elternschaftsabsichten hat (Hypothese 1).

Weiterführend vermittelt die soziale Austauschtheorie, dass soziale Beziehungen nur aufrechterhalten werden, wenn der daraus zu ziehende Nutzen als zufrieden stellend oder zumindest ausreichend eingeschätzt wird. Die Interaktion der Partner (zu verstehen als Austausch von Ressourcen jeglicher Art) führt im Zeitverlauf zu einer Verdichtung und Verstetigung der Beziehung. Dadurch wird die Auflösung der Beziehung immer schwieriger (Schmidt/Moritz 2009: 33f; Hill/Kopp 2004: 104). Darüber hinaus ist die Geburt eines (weiteren) Kindes eine Investition in die bestehende Beziehung. Ein gemeinsames Kind erhöht die Interdependenz der Partner und verlangt zudem eine kontinuierliche Interaktion. Diese Entwicklung wird für die Partner nur wünschenswert sein, wenn die Beziehung bisher Nutzen stiftend war und dies auch für das zukünftige Bestehen der Beziehung angenommen werden kann.

Es ist davon auszugehen, dass Individuen neben ihrem persönlichen Nutzen gleichermaßen das Wohl des zukünftigen Kindes berücksichtigen. Denn dies nimmt ebenfalls Einfluss auf die psychologischen und emotionalen Kosten einer Elternschaft. Demnach werden Individuen bestrebt sein, Konflikte und Trennungen zu vermeiden und ein möglichst optimales Umfeld für das Aufwachsen eines 
Kindes zu schaffen. Gehen Individuen davon aus, diesen hohen Ansprüchen nicht gerecht werden $\mathrm{zu}$ können, so bleibt die Ausbildung eines (weiteren) Kinderwunsches unwahrscheinlich (Peuckert 2008: 114f.). Unter den Gesichtspunkten der Bindungswirkung von Kindern und dem antizipierten Kindeswohl kann demnach angenommen werden,

dass auch eine hohe Partnerschaftsqualität einen positiven Einfluss auf übereinstimmende Elternschaftsabsichten hat (Hypothese 2).

Hypothese 1 und 2 stellen somit zwei konkurrierende Hypothesen dar, welche die potentiell ambivalente Wirkungsweise der Partnerschaftsqualität als Summe verschiedener Aspekte auf den gemeinschaftlichen Kinderwunsch verdeutlichen. Darüber hinaus bestimmen individuelle Erfahrungen, ob eine (weitere) Elternschaft eine Erhöhung oder Verringerung des individuellen bzw. partnerschaftlichen Nutzens erwarten lässt (Borchardt/Stöbel-Richter 2004: 38, Hill/Kopp 2004: 111f.). $\mathrm{Zu}$ diesen individuellen Erfahrungsbereichen zählen u.a. bereits erworbene Elternschafts- und Beziehungserfahrungen, welche sich konsequenter Weise zwischen Eltern und kinderlosen Paaren unterscheiden. Da sich beide Gruppen hinsichtlich ihrer Erfahrungswerte, was eine Elternschaft bedeutet, und damit auch hinsichtlich der Kinderwunschgenese deutlich gegeneinander abgrenzen, scheint ein Vergleich von Eltern und kinderlosen Paaren nur begrenzt möglich. Dennoch bleibt anzunehmen, dass Eltern die Auswirkungen einer Elternschaft auf ihre Partnerschaft besser einschätzen können als Kinderlose und

daher die Partnerschaftsqualität auf übereinstimmende Elternschaftsabsichten von Paaren mit Kindern anders wirkt als auf die übereinstimmenden Elternschaftsabsichten von kinderlosen Paaren (Hypothese 3). 


\section{$4 \quad$ Daten}

Der Einfluss der Partnerschaftsqualität auf den Kinderwunsch von Paaren wird mit den Daten der ersten Welle des „Panel Analysis of Intimate Relationships and Family Dynamics“ (pairfam) untersucht. Das pairfam-Projekt ist eine repräsentative, interdisziplinäre Längsschnittstudie zur Erforschung partnerschaftlicher und familialer Lebensformen in der Bundesrepublik Deutschland, welche im Rahmen einer transregionalen Kooperation der Universitäten Bremen, Chemnitz, Mannheim und München koordiniert und durch die Deutsche Forschungsgemeinschaft gefördert wird. Das pairfam beinhaltet u.a. die Themenschwerpunkte Gestaltung von Paarbeziehungen und Elternschaftsentscheidungen. Ein zentraler Vorzug dieses Datensatzes ist es, dass im Rahmen der Befragung Informationen von beiden Beziehungspartnern erhoben werden. So können Elternschaftsabsichten und Einschätzungen der individuellen Partnerschaftsqualität in einem dyadischen Kontext analysiert werden. Des Weiteren ermöglicht dies, Entscheidungs- und Handlungsprozesse innerhalb der Partnerschaft sowie mögliche Interdependenzen der Partner, ihren Abstimmungsbedarf und ihre strukturelle und wertbezogene Einflussnahme aufeinander in Bezug auf generative Entscheidungen $\mathrm{zu}$ berücksichtigen (Huinink/Feldhaus 2008: 19ff.).

\subsection{Stichprobe}

Für die Generierung der untersuchten Stichprobe wurden aus dem Anker- und zugehörigen Partnerdatensatz verheiratete wie unverheiratete heterosexuelle Paare selektiert, deren Zielpersonen aus den Kohorten 1971 bis 1973 bzw. 1981 bis 1983 stammen. Paare, bei denen zum Zeitpunkt der Befragung eine Schwangerschaft vorlag, sowie Paare, denen es nach eigenen Angaben mit hoher Wahrscheinlichkeit 
nicht möglich ist, auf natürlichem Wege ein Kind zu bekommen, wurden aus der Stichprobe ausgeschlossen. Eine weitere Reduzierung der Stichprobengröße resultierte aus dem Ausschluss von Paaren mit inkonsistenten Angaben zur Beziehungsdauer sowie einem Beziehungsbeginn, der vor dem 11. Lebensjahr eines Partners liegt. Weiterhin konzentriert sich die Stichprobe auf Paare, bei denen die Frau das 46. Lebensjahr noch nicht vollendet hat und somit eine Umsetzung des potentiellen Kinderwunsches aus biologischer Perspektive in den kommenden Jahren noch möglich wäre.

Da der Kinderwunsch in einem erheblichen Maße davon abhängig ist, ob bereits Kinder vorhanden sind und auch die Partnerschaft selbst sowie deren Bewertung durch die Existenz von Kindern beeinflusst wird (Bulatao 1981: 18, 23; Rijken 2009: 58; Eckhard/Klein 2006: 79), wird die Stichprobe weiterhin nach kinderlosen Paaren und Paaren, die bereits Eltern sind, differenziert. Grundlegend für die Unterscheidung von Eltern und kinderlosen Paaren sind für die vorliegende Untersuchung Elternschaftserfahrungen mindestens eines Partners. Diese bestehen, wenn einer der Partner leibliche Kinder hat, ungeachtet dessen, ob diese noch im gleichen Haushalt mit dem Befragten und seinem Partner leben oder nicht. Auf Basis dieses Selektionsmerkmales ergibt sich eine Teilstichprobengröße von 986 kinderlosen Paaren und 1.807 Paaren, die bereits mindestens ein Kind haben.

\section{Operationaliserung und Deskription der untersuchungsrelevanten Variablen \\ 5.1 Operationalisierung des Kinderwunsches}

Es existieren verschiedene Möglichkeiten, den Kinderwunsch zu erheben (Thomson 2001: 5347, Hagewen/Morgan 2005). Neben der idealen und erwarteten (realistischen) Kinderzahl, werden auch zeitnahe Elternschaftsabsichten in der ersten Welle des pairfam erfragt. Während die Konzepte der idealen Kinderzahl 
und der realistisch erwarteten Kinderzahl weitestgehend unabhängig von einer bestehenden Partnerschaft und einem zeitlichen Bezugsrahmen sind, stellt das Konzept zeitnaher Elternschaftsabsichten zur möglichst exakten Erfassung des Kinderwunsches einen zeitlichen Bezug her, in dem der geäußerte Kinderwunsch umgesetzt werden soll. Eine zeitliche Eingrenzung trägt auch dem Umstand Rechnung, dass sich sowohl persönliche Präferenzen als auch (individuelle) Rahmenbedingungen im Zeitverlauf verändern können (Voas 2003: 638). Dies birgt zusätzlich den Vorteil, dass die Befragten die Realisierungschancen ihrer Kinderwünsche besser einschätzen können als bei einer allgemeineren Frageformulierung (Ette/Ruckdeschel 2007: 58). Das Konzept zeitnaher Elternschaftsabsichten enthält nicht nur einen zeitlichen Rahmen für die Umsetzung von Kinderwünschen, sondern berücksichtigt den Partnerschaftskontext, indem nur Personen, die sich aktuell in einer Partnerschaft befinden, danach gefragt werden. Die Analyse von Kinderwünschen in diesem Beitrag bezieht sich daher auf die Übereinstimmung der Partner bei ihren zeitnahen Elternschaftsabsichten. Frauen und Männer, die sich in einer Paarbeziehung befinden, wurden gefragt, ob sie vorhaben, in den nächsten zwei Jahren Mutter bzw. Vater zu werden.

Tabelle 1 dokumentiert die Übereinstimmung der Antworten auf diese Frage. Dabei zeigt sich, dass sowohl kinderlose Paare als auch Elternpaare mehrheitlich übereinstimmende Elternschaftsabsichten haben. Von den Elternpaaren geben 66,4 Prozent an, in den nächsten zwei Jahren kein weiteres Kind bekommen zu wollen. Dies trifft nur auf 36,2 Prozent der kinderlosen Paare zu. Kinderlose Paare weisen hingegen deutlich häufiger als Elternpaare den übereinstimmenden Wunsch auf, Eltern zu werden. Allerdings herrscht unter kinderlosen Paaren auch häufiger Uneinigkeit über die Elternschaftsabsicht. Betrachtet man diese divergierenden Elternschaftsabsichten, zeigt sich, dass bei Eltern wie Kinderlosen weder Mann 
noch Frau eine stärkere Tendenz zum Wunsch nach einem (weiteren) Kind aufweisen.

[Tabelle 1]

\subsection{Operationalisierung der Partnerschaftsqualität}

Zur Bewertung der Partnerschaftsqualität existieren keine objektiven Kriterien (Hahlweg/Baucom 2008: 3). Die Messung erfolgt vorrangig über die subjektive Einschätzung verschiedener Partnerschaftsbereiche (z.B. Partnerschaftszufriedenheit, positive und negative Interaktion) durch den Befragten. Dies verdeutlicht, dass das Konstrukt der Partnerschaftsqualität über verschiedene Dimensionen gemessen wird, die mit positiven bzw. negativen Facetten einer Beziehung assoziiert werden. Das pairfam bietet dazu ein breites Spektrum an relevanten Fragen. Für die Untersuchung des potenziell ambivalenten Einflusses der subjektiven Partnerschaftsqualität auf den Kinderwunsch werden vier Aspekte der Partnerschaftsqualität herangezogen, um diese möglichst umfassend abzubilden. Die subjektive Beziehungszufriedenheit und das wahrgenommene Ausmaß positiver Interaktion in der Partnerschaft gelten als Indikatoren für den positiven Einfluss einer hohen Partnerschaftsqualität auf den Kinderwunsch. Die Trennungsabsicht und die berichtete Häufigkeit negativer Interaktion in der Beziehung dienen hingegen zur Untersuchung des positiven Einflusses einer geringen Partnerschaftsqualität auf die Elternschaftsabsichten. Diese evaluativen Partnerschaftsaspekte wurden für die anschließenden Analysen wie folgt operationalisiert: 
Die subjektive Beziehungszufriedenheit wird im pairfam über die Formulierung „Wie zufrieden sind Sie insgesamt mit Ihrer Beziehung?“ erfragt. Diese kann von den Befragten mit Hilfe einer 11-stufigen Skala beantwortet werden, die von ,sehr unzufrieden“ (0) bis „sehr zufrieden“ (10) reicht. Für die Auswertung der Partnerschaftszufriedenheit und nachfolgende Analysen wurde aus dieser Skala eine drei Kategorien umfassende Variable generiert. Aufgrund des allgemein hohen $\mathrm{Zu}$ friedenheitsniveaus wurden die Skalenwerte 9 und 10 zur Kategorie „sehr zufrieden“ und die Werte 7 und 8 zur Kategorie „zufrieden“ zusammengefasst. Personen, welche die Zufriedenheit mit ihrer Beziehung mit einem Wert 0 bis 6 bewerteten, wurden der neu generierten Kategorie ,weniger zufrieden bis sehr unzufrieden" zugeordnet.

Die Trennungsabsicht wird im pairfam individuell über die Frage „Haben Sie im letzten Jahr ernsthaft an eine mögliche Trennung oder Scheidung gedacht?“ erhoben und kann von den Befragten mit ja oder nein beantwortet werden. Diese Kategorien wurden für die folgenden Analysen beibehalten.

Das Ausmaß positiver Interaktion in der Partnerschaft wird über eine Auswahl von zehn Items operationalisiert, die mit einer positiven Interaktion beider Partner assoziiert werden:

1. „Ich gebe meinem Partner/meiner Partnerin das Gefühl, dass ich ihn/sie verstehe. “

2. „Ich höre meinem Partner/meiner Partnerin zu, gebe ihm/ihr Raum, sich auszusprechen."

3. „Ich unterstütze meinem Partner/meiner Partnerin mit Rat und Tat, wenn er/sie Probleme hat. "

4. „Mein Partner/meine Partnerin gibt mir das Gefühl, dass er/sie mich versteht.“

5. „Mein Partner/meine Partnerin hört mir zu, gibt mir Raum, mich auszusprechen. “

6. „Mein Partner/meine Partnerin unterstützt mich mit Rat und Tat, wenn ich Probleme habe."

7. „Wie oft erzählen Sie Ihrem Partner/Ihrer Partnerin was Sie beschäftigt?“

8. „Wie oft zeigt Ihr Partner/Ihre Partnerin Ihnen Anerkennung für das, was Sie tun?“

9. „Wie oft zeigt Ihnen Ihr Partner/Ihre Partnerin, dass er/sie Sie schätzt?

10. „Wie oft teilen Sie mit Ihrem Partner/Ihrer Partnerin Ihre Geheimnisse und innersten Gefühle? “ 
Die Befragten konnten bei diesen Fragen bzw. Aussagen zwischen den Antwortvorgaben „nie“(1), „selten“ (2), „,manchmal“ (3), „häufig“ (4) und „immer“ (5) wählen. Aus diesen fünf Kategorien wurde eine Skala zur Bemessung der erfahrenen Häufigkeit positiver Interaktion in der Partnerschaft erstellt, wobei Befragte, die keine Angaben machten oder die Antwortvorgabe „weiß nicht“ wählten, ausgeschlossen wurden. Die resultierende Skala wurde in drei Kategorien rekodiert, die aussagen, ob Befragte das Ausmaß der positiven Interaktion in ihrer Partnerschaft als „,niedrig“, ,mittel“ oder „,hoch“ bewerten. Für die nachfolgenden Analysen wurden die Kategorien „niedrig“ und „mittel“ aufgrund der darin enthaltenen geringen Fallzahl $\mathrm{zu}$ der Kategorie „niedrig bis mittel“ zusammengefasst. Alle in die Erstellung der Skala einbezogenen Items waren positiv miteinander korreliert und Cronbach's Alpha mit einem Wert von 0,857 für Männer und einem Wert von 0,864 für Frauen ausreichend hoch, um zur Bestätigung der Reliabilität akzeptiert zu werden.

Eine negative Interaktion wurde über die subjektive Einschätzung der Häufigkeit von negativem Konfliktverhalten in der Partnerschaft gemessen. Dabei wurde erfragt, wie häufig die folgenden Verhaltensweisen bei auftretenden Meinungsverschiedenheiten vom Befragten selbst bzw. seinem Partner gezeigt werden:

1. „Den Partner beleidigen oder beschimpfen“

2. „In Schweigen verfallen”

3. "Den Partner anschreien"

4. "Sich weigern, weiter darüber zu reden"

5. „Schnell beleidigt sein”

6. „Dem anderen die Schuld geben, Schuldgefühle machen“

Die Befragten konnten diese Frage anhand einer Skala beantworten, die von ,fast nie oder nie“ (1) bis „sehr oft“(5) reichte. Zur Bemessung der Dimension des 
negativen Konfliktverhaltens in der Partnerschaft wurde aus dem Antwortverhalten eine Skala erstellt, welche die drei Kategorien „, selten bis nie“ (1), „manchmal“ (2) und „,häufig bis immer“ (3) umfasst. Aufgrund zu geringer Häufigkeiten wurden die Kategorien (1) und (2) für die nachfolgenden Analysen $\mathrm{zu}$ der Kategorie „gelegentlich bis häufig“ zusammengefasst. Personen, die keine Angaben machten oder sich der Kategorie „, weiß nicht“ zuordneten, flossen nicht in die Erstellung der Skala mit ein. Auch in diesem Fall sind alle Items positiv miteinander korreliert und der Wert von Cronbach's Alpha mit 0,870 für Männer und 0,852 für Frauen ausreichend hoch, um zur Bestätigung der Reliabilität akzeptiert zu werden.

[Tabelle 2]

Tabelle 2 gibt die Einschätzung der Partnerschaftsqualität getrennt nach kinderlosen Paaren und Eltern wieder. Es zeigt sich, dass die Bewertung der Partnerschaftsqualität beider Partner, sowohl bei kinderlosen Paaren als auch bei Elternpaaren, größtenteils übereinstimmt. Nur bei Betrachtung der positiven Interaktion wird deutlich, dass Eltern häufiger als Kinderlose keine übereinstimmende Bewertung aufweisen. Zudem bescheinigen kinderlose Paare ihrer Beziehung eine höhere Qualität als Eltern: Paare, die (noch) keine Kinder haben, sind häufiger sehr zufrieden mit ihrer Beziehung, nehmen häufiger positive Interaktion in der Partnerschaft wahr und berichten gleichzeitig über ein geringeres Ausmaß an negativem Konfliktverhalten. Die geringere Trennungsabsicht von Eltern könnte auf die Bindungswirkung bereits existierender Kinder zurückzuführen sein (Peuckert 2005: 185; Klein/Eckhard 2008: 379f.). 
Basis der empirischen Analysen ist ein logistisches Regressionsmodell. In diesem Modell ergibt die abhängige Variable 1, wenn sich beide Partner ein Kind wünschen und 0 wenn dies nicht zutrifft (dies schließt auch unterschiedliche Meinungen der Partner ein).

Dem aktuellen Forschungsstand ist zu entnehmen, dass Eltern und kinderlose Paare unterschiedliche Erfahrungshorizonte in Bezug auf Elternschaftserfahrungen aufweisen. Diese Erfahrungen können wiederum einen Einfluss auf die Ausbildung eines (weiteren) Kinderwunsches haben. Dies führt dazu, dass die Elternschaftsabsichten beider Gruppen nicht direkt miteinander vergleichbar sind und es sich daher nicht anbietet, Eltern und Kinderlose in einem Modell zu belassen.

Konsequenterweise wurden daher für Eltern und kinderlose Paare separate binäre logistische Regressionen (d.h. auf gruppierten Daten basierte LogitRegressionsmodelle) berechnet (Litz 2000: 169). Aus dem gegenwärtigen Forschungsstand geht zudem hervor, dass geschlechtsspezifische Unterschiede bei der Wahrnehmung von Partnerschaftsqualität und der Ausbildung von Elternschaftsabsichten bestehen. Getrennte Modelle für Frauen und Männer sollen es ermöglichen, den Einfluss einzelner Merkmale von Männern und Frauen auf den gemeinsamen Kinderwunsch zu berücksichtigen. ${ }^{1}$ Dabei wurden die unabhängigen Variablen (siehe Tabelle A1) stufenweise in die einzelnen Logit-Modelle integriert. Die in den folgenden Abschnitten aufgeführten Modelle enthalten nur jene

\footnotetext{
${ }^{1}$ Zur Analyse des dyadischen Kinderwunsches würde man idealer Weise beide Partner in ein gemeinsames Modell integrieren. Durch die Fülle der relevanten Kontrollvariablen würde ein solches Vorgehen zu einer Überspezifizierung des Modells führen. Eine Aussage über Einflussfaktoren, die tatsächlich einen Einfluss auf die Ausbildung des dyadischen Kinderwunsches haben, wäre damit nicht mehr möglich.
} 
unabhängigen Variablen, die zur höchsten Modellgüte (gemessen am LogLikelihood-Ratio-Test) beitragen.

Da die Kreuzkorrelationen zwischen den verschiedenen Dimensionen der Partnerschaftsqualität hoch sind (die Werte für Cronbach's Alpha liegen zwischen 0,852 und 0,870), wurden separate Modelle für die vier Aspekte der Partnerschaftsqualität (individuelle Beziehungszufriedenheit, positive Interaktion, Trennungsabsicht, negative Interaktion) berechnet.

Aus diesen Differenzierungen ergeben sich für die Analyse des übereinstimmenden Kinderwunsches die Modelle $3 \mathrm{a}$ bis $3 \mathrm{~h}$ sowie $4 \mathrm{a}$ bis $4 \mathrm{~h}$ (siehe Tabelle 3 und 4). Durch die Berechnung separater Modelle wird ein Vergleich der übereinstimmenden Elternschaftsabsichten von Eltern und kinderlosen Paaren unter Berücksichtigung geschlechtsspezifischer Unterschiede möglich. So kann untersucht werden, wie sich die Merkmale von Müttern und Vätern bzw. von kinderlosen Frauen und Männern auf den gemeinsamen Kinderwunsch auswirken.

\section{$7 \quad$ Ergebnisse}

Beim Vergleich der beiden Teilstichproben von Eltern und kinderlosen Paaren (Tabelle 3 und 4) wird deutlich, dass wir unsere Forschungsfrage zu möglichen Unterschieden zwischen Eltern und kinderlosen Paaren hinsichtlich des übereinstimmenden Kinderwunsches dahingehend beantworten können, dass systematische Unterschiede zwischen beiden Gruppen bestehen. Bei Paaren, die bereits Kinder haben, zeigt sich, dass der übereinstimmende Wunsch nach einem weiteren Kind stärker vom individuellen Lebensalter abhängig ist als bei kinderlosen Paaren. Befinden sich die kinderlosen Partner in einem jungen Alter (unter 35 Jahren) oder nahe dem Ende ihrer fertilen Phase, sinkt die Wahrscheinlichkeit für übereinstimmende Elternschaftsabsichten signifikant (vgl. 
Modelle 3a bis 3h). Für Eltern zeigt sich hingegen, dass der übereinstimmende Wunsch nach einem weiteren Kind mit zunehmendem Lebensalter unwahrscheinlicher wird (vgl. Modelle 4a bis 4h). Dabei ist vor allem das Alter der Frau von signifikanter Bedeutung. Sind Frauen jünger als 30 Jahre, werden übereinstimmende Wünsche der Eltern nach einem weiteren Kind deutlich wahrscheinlicher.

Die Herkunft der Partner ist vorrangig für die Elternschaftsabsichten bislang kinderloser Paare von Bedeutung. Übereinstimmende Wünsche nach einem Kind sind bei kinderlosen Paaren eher zu erwarten, wenn einer der Partner in der ehemaligen DDR geboren wurde (vgl. Modelle 3a bis 3h). Für den Wunsch nach einem weiteren Kind ist die regionale Herkunft kaum relevant. Ein leicht positiver Einfluss auf den gemeinsamen Kinderwunsch geht lediglich von aus dem Ausland stammenden Frauen, die bereits Kinder haben, aus (vgl. Modelle 3e und 3g).

Das erzielte Bildungsniveau beider Partner hat keinen Einfluss auf die Fertilitätsabsichten von kinderlosen Paaren (vgl. Modelle 3a bis 3h). Für Eltern erhöht sich die Wahrscheinlichkeit, dass sich beide Partner ein weiteres Kind wünschen, wenn beide die (Fach-)Hochschulreife oder das Abitur erzielt haben (vgl. Modelle 4a bis 4h). Der Erwerbsstatus der Partner steht weder bei kinderlosen Paaren noch bei Paaren mit Kindern in einem Zusammenhang mit deren übereinstimmenden zeitnahen Elternschaftsabsichten (vgl. Modelle 3a bis 4h). Hier konnte weder für Eltern noch für kinderlose Paare ein signifikanter Zusammenhang gefunden werden.

Der Einfluss der religiösen Überzeugung auf den Kinderwunsch stellt sich für die untersuchten Stichproben etwas differenzierter dar. Bei kinderlosen Paaren fördert die Religiosität des Mannes den übereinstimmenden Wunsch nach einem ersten Kind (vgl. Modelle 3a bis 3d). Dieser Einfluss zeigt sich für Elternpaare weniger 
deutlich (vgl. Modelle 4a bis 4d). Jedoch konnte ein deutlich positiver Effekt der Religionszugehörigkeit der Frau auf den übereinstimmenden partnerschaftlichen Wunsch nach einem weiteren Kind gezeigt werden (vgl. Modelle 4e bis 4h).

Unterschiede zwischen kinderlosen Paaren und Paaren mit Kindern werden deutlich, wenn der Institutionalisierungsgrad der Partnerschaft als Determinante der gemeinsamen Fertilitätsabsicht herangezogen wird. Der Wunsch nach einem weiteren Kind bleibt bei Eltern vom Institutionalisierungsgrad der Partnerschaft unbeeinflusst (vgl. Modelle 4a bis 4h). Bei kinderlosen Paaren sinkt die Wahrscheinlichkeit eines übereinstimmenden Kinderwunsches, je geringer der Grad der Institutionalisierung der Beziehung ist (vgl. Modelle 3a bis 3h). D.h. kinderlose Paare, die in einer nichtehelichen Lebensgemeinschaft leben, haben seltener übereinstimmende Elternschaftsabsichten als verheiratete Paare ohne Kinder.

Werden bereits vorhandene Kinder in den Modellen berücksichtigt, zeigt sich dass Elternschaftsabsichten in einem starken Maße von bereits vorhandenen Kindern abhängen. Demnach wird ein gemeinsamer Wunsch von Eltern nach einem weiteren Kind mit zunehmender Kinderzahl unwahrscheinlicher (vgl. Modelle 4a bis 4h). Dies verdeutlicht, dass die Existenz von Kindern zu einer unterschiedlichen Wirkung der Kontrollvariablen auf den übereinstimmenden Kinderwunsch führt. Geht der Einfluss von Kindern über eine Wirkung auf strukturelle Merkmale der Partnerschaft hinaus, müsste sich dies gleichermaßen in Unterschieden beim Einfluss der Partnerschaftsqualität auf die Elternschaftsabsichten von Eltern und kinderlosen Paaren niederschlagen.

[Tabelle 3]

[Tabelle 4] 
Hinsichtlich unserer zweiten zentralen Forschungsfrage zur Wirkungsweise der Partnerschaftsqualität auf den Kinderwunsch wird deutlich, dass einzelne Aspekte der Partnerschaftsqualität unterschiedlichen Einfluss auf die gemeinsamen Elternschaftsabsichten von Kinderlosen und Eltern haben. Bei Betrachtung der subjektiven Beziehungszufriedenheit kinderloser Paare zeigt sich, dass nur eine sehr hohe Zufriedenheit des Mannes einen signifikant positiven Einfluss auf die gemeinsamen Elternschaftsabsichten hat (vgl. Modelle 3a und 3e). Hinsichtlich der Elterngruppe wird deutlich, dass eine sehr hohe Beziehungszufriedenheit der Frau einen positiven Effekt auf den gemeinsamen Kinderwunsch hat. Eine geringe Beziehungszufriedenheit des Mannes hat hingegen einen negativen Effekt auf den gemeinsamen Wunsch nach weiteren Kindern (vgl. Modelle 4a und 4e). Damit überwiegt der positive Einfluss der subjektiven Beziehungszufriedenheit auf die übereinstimmenden Elternschaftsabsichten.

Die Wahrnehmung positiver Interaktion in der Partnerschaft steht ebenfalls in keinem signifikanten Zusammenhang mit den Elternschaftsabsichten von kinderlosen Paaren (vgl. Modelle 3c und 3g). Nimmt hingegen ein Mann, der bereits Vater ist, erhebliche positive Interaktion in der Beziehung wahr, sinkt die Wahrscheinlichkeit eines übereinstimmenden Wunsches des Paares nach einem weiteren Kind (vgl. Modell 4c). Die Wahrnehmung positiver Interaktion von Müttern steht in keinem signifikanten Zusammenhang mit dem gemeinschaftlichen Kinderwunsch (vgl. Modell 4g).

Deutliche Unterschiede zwischen den Elternschaftsabsichten beider Paargruppen sind bei der Untersuchung des Einflusses der Trennungsabsicht zu erkennen. Diese steht lediglich mit dem Kinderwunsch von Eltern in einem signifikant negativen Zusammenhang. Hat einer der Partner im letzten Jahr ernsthaft über eine Trennung nachgedacht, sinkt die Chance, dass sich beide Partner in den nächsten zwei Jahren 
ein weiteres Kind wünschen, um fast die Hälfte (vgl. Modelle 4b und 4f). Eine ausgeprägte Trennungsabsicht hat somit einen negativen Effekt auf gemeinsame Elternschaftsabsichten von Paaren.

Ähnliches geht aus der Einbeziehung der Häufigkeit negativer Interaktion hervor. Für kinderlose Paare ist kein signifikanter Einfluss nachweisbar (vgl. Modelle 3d und 3h). Tritt negatives Konfliktverhalten bei Paaren mit Kindern nur selten auf, ist die Wahrscheinlichkeit für einen von den Partnern geteilten Wunsch nach einem weiteren Kind signifikant erhöht (vgl. Modelle 4d und 4h). Damit wurde ein nachteiliger Effekt negativer Interaktion auf gemeinsame Elternschaftsabsichten bestätigt.

Zusammenfassend verdeutlichen die den Modellen $\mathrm{zu}$ entnehmenden Untersuchungsergebnisse somit, dass zwischen den partnerschaftlichen Elternschaftsabsichten von Eltern und Kinderlosen Unterschiede bestehen. Diese äußern sich vornehmlich beim Einfluss des Alters, der Herkunft und der Religiosität der Partner, aber auch durch den Institutionalisierungsgrad der Beziehung. Aus den Merkmalen Alter und Religiosität geht darüber hinaus hervor, dass die Charakteristiken von Männern und Frauen bzw. Vätern und Müttern in unterschiedlicher Weise auf deren gemeinsame Elternschaftsabsichten wirken. Gemäß diesen systematischen Unterschieden zwischen Eltern und kinderlosen Paaren hinsichtlich der Ausbildung ihrer übereinstimmenden Kinderwünsche, muss Hypothese 3, zur unterschiedlichen Wirkungsweise der Partnerschaftsqualität auf die generativen Absichten von Eltern und Kinderlosen, angenommen werden.

Bei der Untersuchung der Wirkung einzelner Partnerschaftsaspekte wird deutlich, dass die Beziehungsqualität lediglich für die generative Entscheidung von Paaren, die bereits Kinder haben, eine signifikante Bedeutung hat. Für Eltern wirkt eine hohe Partnerschaftsqualität, gemessen anhand der subjektiven 
Beziehungszufriedenheit und einer positiven Interaktion, positiv auf gemeinsame Elternschaftsabsichten. Eine niedrige Beziehungszufriedenheit, evaluiert durch eine Trennungsabsicht und negative Interaktion zwischen den Partnern, hat hingegen einen negativen Einfluss auf gemeinsame Elternschaftsabsichten. Demnach kann Hypothese 2, zur positiven Wirkungsweise einer hohen Partnerschaftsqualität auf den übereinstimmenden Kinderwunsch, zumindest für Eltern, angenommen werden. Hypothese 1, zum positiven Einfluss einer geringen Partnerschaftsqualität auf übereinstimmende Elternschaftsabsichten, ist folglich abzulehnen.

\section{Diskussion und Ausblick}

Im Fokus dieser Untersuchung stand der Einfluss der Partnerschaftsqualität auf den übereinstimmenden Kinderwunsch von Paaren. In der Betrachtung der Paarperspektive, die durch das Erhebungsdesign des pairfam und der daraus resultierenden Befragung beider Partner möglich wird, liegt die Besonderheit dieses Beitrags.

Als erstes zentrales Ergebnis dieser Untersuchung seien die homogenen zeitnahen Kinderwünsche von Eltern wie kinderlosen Paaren genannt. Lediglich 26 Prozent der kinderlosen Paare sowie 13 Prozent der Elternpaare weisen divergierende Elternschaftsabsichten auf (Vgl. Tabelle 1). Diese Zahlen verdeutlichen, dass vor allem Paare mit Elternschaftserfahrungen eine hohe Übereinstimmung hinsichtlich ihrer kurzfristigen Elternschaftsabsichten äußern.

Als zweiter zentraler Befund stellt sich der Einfluss der Partnerschaftsqualität auf die übereinstimmenden Elternschaftsabsichten dar. So wurde deutlich, dass die Wahrnehmung der Partnerschaftsqualität lediglich mit der generativen Entscheidung von Eltern in einem signifikanten Zusammenhang steht. Die Wahrnehmung der untersuchten Qualitätsaspekte der Beziehung ist für den 
Kinderwunsch von kinderlosen Paaren hingegen kaum relevant. Bei kinderlosen Paaren nimmt die Wahrnehmung der untersuchten Qualitätsaspekte der Beziehung kaum einen signifikanten Einfluss auf ihre generativen Absichten. Bei Eltern können hingegen eine hohe Beziehungszufriedenheit und ein selten auftretendes negatives Konfliktverhalten in der Partnerschaft den Wunsch nach einem weiteren Kind fördern. Eine Trennungsabsicht wirkt hingegen negativ auf Elternschaftsabsichten. Demnach hat eine hohe Partnerschaftsqualität einen positiven Einfluss auf den von beiden Partnern geteilten Wunsch nach einem weiteren Kind, dies gilt zumindest für Paare mit vorhandenen Elternschaftserfahrungen. Es ist anzunehmen, dass Eltern ihre generative Entscheidung stärker von der wahrgenommenen Beziehungsqualität abhängig machen, da sie bereits wissen, welche tatsächlichen Auswirkungen eine Elternschaft auf ihre Partnerschaft hat. Kinderlose Paare haben womöglich eine stärker idealisierte Vorstellung von der Geburt ihres ersten Kindes. Da Eltern eine geringere Partnerschaftsqualität berichten als kinderlose Paare, könnten Eltern bewusst mit weiteren Einbußen in ihrer Beziehungsqualität durch die Geburt eines weiteren Kindes rechnen und sich daher nur bei einer sehr hohen Partnerschaftsqualität für ein weiteres Kind entscheiden. Der Umkehrschluss, dass auch eine geringe Partnerschaftsqualität den Elternschaftsabsichten von Paaren förderlich sein könnte, kann mit den vorliegenden Ergebnissen weder bestätigt noch verworfen werden.

Die vorliegenden Ergebnisse basieren auf Querschnittsdaten der ersten Welle des paifam. Daher können mit dieser Studie keine Aussagen zur kausalen Wirkung von Partnerschaftsqualität auf den Kinderwunsch von Paaren getroffen werden. Damit bleibt offen, ob der weitere Kinderwunsch aus einer hohen Partnerschaftsqualität der Eltern resultiert oder ob die Partnerschaftsqualität hoch ist, weil ein 
gemeinsamer Kinderwunsch vorhanden ist. Mit der Verwendung von Querschnittsdaten muss auch die Frage offen bleiben, ob Eltern und kinderlose Paare unterschiedliche Phasen im individuellen Lebensverlauf darstellen oder ob es sich in der Tat um gänzlich verschiedene Personengruppen handelt. Weitere Erhebungswellen des pairfam können zu einer Klärung dieser Fragen beitragen. Gleiches gilt auch für die Fragen, ob eine hohe subjektive Partnerschaftsqualität eher $\mathrm{zu}$ einer Umsetzung von Kinderwünschen führt und $\mathrm{zu}$ wessen Gunsten divergierende Elternschaftsabsichten letztlich umgesetzt werden. Dies könnte Aufschluss darüber geben, ob die Elternschaftsentscheidung tatsächlich ein dyadischer Aushandlungsprozess ist und wenn ja, welchem Partner innerhalb einer Beziehung eine stärkere Entscheidungsmacht bei der generativen Entscheidung zukommt. 
BeCKMAN, L.J. (1977): Couples’ Decision-Making Process Regarding Fertility. In: Taeuber, K.E./Bumpass, L.L./Sweet, J.A. (ed.): Social Demography. New York: Academic Press.

Beckman, L.J. (1982): Measuring the Process of Fertility Decision-Making. In: Fox, G.L. (ed.): The Childbearing Decision: Fertility Attitudes and Behavior. Beverly Hills: Sage Publications.

BorCHARDT, A./STÖBEL-RICHTER, Y. (2004): Die Genese des Kinderwunsches bei Paaren - eine qualitative Studie. In: Materialien zur Bevölkerungswissenschaft, Heft 114. Wiesbaden: Bundesinstitut für Bevölkerungsforschung.

BulataO, R.A. (1981): Values and Disvalues of Children in Successive Childbearing Decisions. In: Demography, 18(1): 1-25.

ECKHARD, J./KLEIN, T. (2006): Männer, Kinderwunsch und generatives Verhalten. Eine Auswertung des Familiensurvey zu Geschlechterunterschieden in der Motivation zur Elternschaft. Wiesbaden: VS Verlag für Sozialwissenschaften.

ECKHARD, J./KLEIN, T. (2007): Die Motivation zur Elternschaft. Unterschiede zwischen Männern und Frauen. In: Konietzka, D./Kreyenfeld, M. (Hrsg.): Ein Leben ohne Kinder: Kinderlosigkeit in Deutschland. Wiesbaden: VS Verlag für Sozialwissenschaften.

ENGELHARD, H. (2004): Fertility intentions and preferences: effects of structural and financial incentives and constraints in Austria. Vienna Institute of Demography, Vienna 2004, 23 p. (Working papers 2/2004).

ETTE, A./RUCKDESCHEL, K. (2007): Die Oma macht den Unterschied! Der Einfluss institutioneller und Informeller Unterstützung für Eltern auf ihre weiteren Kinderwünsche. In: Zeitschrift für Bevölkerungswissenschaft, 32(1-2): 51-72.

GRAF, J. (2002): Wenn Paare Eltern werden. Weinheim: Verlagsgruppe Beltz.

HAGEWEN, K.J./MORGAN, S.P. (2005): "Intended and ideal family size in the United States, 19702002." Population and Development Review 31(3): 507-527.

HAYFORD, S.R. (2009): Evolution of Fertility Expectations over the Life Course. Demography 46(4): 765-783.

HAHLWEG/BAUCOM (2008): Partnerschaft und psychische Störungen. Fortschritte der

Psychotherapie, Band 34. Göttingen: Hogrefe, Verlag,.

HEILAND , F,/Prskawetz, A. /Sanderson, W. C. (2008). Are individuals' desired family sizes stable? Evidence from West German panel data. European Journal of Population, 24, 129-156.

HILL, P.B./KoPP, J. (2004): Familiensoziologie: Grundlagen und theoretische Perspektiven. 3., überarbeitete Auflage. Wiesbaden: VS Verlag für Sozialwissenschaften.

HoffMAN, L.W./HofFMAn, M.L. (1973): The Value of Children to Parents. In: Fawcett, J.T. (ed.): Psychological perspectives on population, New York: Basic Books.

HÖHN, C./ETTE, A./RuCKDESCHEL, K. (2006): Kinderwünsche in Deutschland: Konsequenzen für eine nachhaltige Familienpolitik. Stuttgart: Robert-Bosch-Stiftung/Bundesinstitut für Bevölkerungsforschung.

HuininK, J./FELDHAUS, M. (Hrsg.) (2008): Neuere Entwicklungen in der Beziehungs- und Familienforschung. Vorstudien zum Beziehungs- und Familienentwicklungspanel (PAIRFAM). Würzburg: Ergon Verlag.

HuInINK, J./KoniETZKA, D. (2007): Familiensoziologie: eine Einführung. Frankfurt/Main: Campus Verlag.

JOKELA, M. (2010): Characteristics of the first child predict parent's probability of having another child. Developmental Psychology, 2010 (46): 915-926

KlaUS, D./SucKOW, J. (2006): Der Wert von Kindern und sein langer Schatten. Eine kritische Würdigung der VOC-Forschung. In: Steinbach, A. (Hrsg.): Generatives Verhalten und Generationenbeziehungen. Festschrift für Bernhard Nauck zum 60. Geburtstag. Wiesbaden: VS Verlag für Sozialwissenschaften.

KLEIN, T./ECKHARD, J. (2008): Partnerschafts- und berufsbezogene Aspekte des Kinderwunsches von Männern und Frauen. In: Feldhaus, M./Huinink, J. (Hrsg.): Neuere Entwicklungen in der Beziehungs- und Familienforschung. Vorstudien zum Beziehungs- und Familienentwicklungspanel (PAIRFAM). Würzburg: Ergon Verlag.

LENZ, K. (2009): Soziologie der Zweierbeziehung. Eine Einführung. 4. Auflage. Wiesbaden: VS Verlag für Sozialwissenschaften.

LILLARD, L.A./WAITE, L.J. (1993): A Joint of Marital Childbearing and Marital Disruption. In: Demography, 30(4): 653-681.

LITZ, H.P. (2000): Multivariate Statistische Methoden und ihre Anwendung in den Wirtschafts- und Sozialwissenschaften. München: Oldenbourg Wissenschaftsverlag.

MEYERS, S.M. (1997): Marital uncertainty and childbearing. Social Forces, 75, 1271-1289. 
NAUCK, B. (2001): Der Wert von Kindern für ihre Eltern. „Value of Children“ als spezielle Handlungstheorie des generativen Verhaltens und von Generationenbeziehungen im interkulturellen Vergleich. In: Kölner Zeitschrift für Soziologie und Sozialpsychologie, 53(3): 407-435.

PEUCKERT, R. (2008): Familienformen im sozialen Wandel. 7., vollständig überarbeitete Auflage. Wiesbaden: VS Verlag für Sozialwissenschaften.

RIJKEN, A.J. (2009): Happy families, high fertility? Childbearing choices in the context of family and Partner relationships. Dissertation Utrecht University.

RiJKen, A.J./LiEfBROER, A.C. (2009): The Influence of Partner Relationship Quality on Fertility. In: European Journal of Population, 25: 487-502.

RiJKEN, A.J./ThOMSON, E. (2010): Partners' relationship quality and childbearing. In: Social Sci. Res. (2010), doi:10.1016/j.ssresearch.2010.10.001

RosinA, A./TestA, M.R. (2009): Couples' First Child Intentions and Disagreement: An Analysis of the Italian case. In: European Journal of Population, 25: 487-502.

SCHMIDT, U./MORITZ, M.-T. (2009): Familiensoziologie. Bielefeld: transcript Verlag.

ThiBAut, J.W./KelleY, H.H. (1959): The Social Psychology of Groups. New York: Wiley.

Thomson, E. (2001): Family Size Preferences. In: Smelser, N.J./Baltes, P.B. (ed.): International Encyclopedia of the Social \& Behavioral Sciences: 5347-5350.

VOAS, D. (2003): Conflicting Preferences: A Reason Fertility Tends to Be Too High or Too Low. In: Population and Development Review, 29(4): 627-646. 
Tabelle A1 Deskriptive Angaben der logit-Modell-Variablen

\begin{tabular}{|c|c|c|c|c|}
\hline & Männer & Frauen & Männer & Frauen \\
\hline & \multicolumn{2}{|c|}{ kinderlos } & \multicolumn{2}{|c|}{ mit Kindern } \\
\hline & $\%$ & $\%$ & $\%$ & $\%$ \\
\hline \multicolumn{5}{|l|}{ Alter } \\
\hline unter 25 & 2,8 & 19,6 & 0,7 & 3,1 \\
\hline 25 bis 29 & 58,1 & 60,1 & 15,6 & 25,1 \\
\hline 30 bis 34 & 11,8 & 5,7 & 9 & 13 \\
\hline 35 bis 39 & 20,8 & 13,6 & 53,7 & 56,1 \\
\hline 40 und älter & 6,5 & 1 & 21 & 2,7 \\
\hline \multicolumn{5}{|l|}{ Herkunft } \\
\hline $\mathrm{BRD}$ & 73 & 71,9 & 62,5 & 60,7 \\
\hline DDR & 19,5 & 19 & 22,4 & 23,2 \\
\hline Ausland & 7,5 & 9,1 & 15,1 & 16 \\
\hline keine Angabe & & & & 0,1 \\
\hline \multicolumn{5}{|l|}{ Bildungsniveau } \\
\hline \multicolumn{5}{|l|}{ kein Abschluss/ } \\
\hline Hauptschulabschluss* & 15,6 & 8,7 & 26,6 & 18,6 \\
\hline Realschulabschluss & 32,3 & 30,8 & 36,6 & 43,9 \\
\hline \multicolumn{5}{|l|}{ Abitur/(Fach-) } \\
\hline Hochschulreife** & 49,9 & 58 & 33,8 & 35,1 \\
\hline sonstiger Abschluss & 2,2 & 2,4 & 3 & 2,4 \\
\hline \multicolumn{5}{|l|}{ Erwerbsstatus } \\
\hline nicht erwerbstätig & 6,1 & 5,8 & 7,1 & 43,6 \\
\hline in Ausbildung & 19 & 25,7 & 3,8 & 4,8 \\
\hline teilzeitbeschäftigt & 3,7 & 9,2 & 3,9 & 36,9 \\
\hline voll erwerbstätig & 71,2 & 59,3 & 84,9 & 14,5 \\
\hline keine Angabe & 0,1 & & 0,3 & 0,2 \\
\hline \multicolumn{5}{|l|}{ Religiosität } \\
\hline sehr religiös & 6 & 6,8 & 10,6 & 13,2 \\
\hline religiös & 10,5 & 13,2 & 15,3 & 20,4 \\
\hline weniger religiös & 31,1 & 33,4 & 27,8 & 27 \\
\hline gar nicht religiös & 51,2 & 44,3 & 44,8 & 38,6 \\
\hline keine Angabe & 1,1 & 2,3 & 1,5 & 0,9 \\
\hline \multicolumn{5}{|c|}{ Institutionalisierungsgrad der Beziehung } \\
\hline \multicolumn{5}{|c|}{$\begin{array}{l}\text { NEL ohne } \\
\text { gemeinsamen }\end{array}$} \\
\hline \multicolumn{5}{|l|}{ NEL mit } \\
\hline Haushalt & 47,4 & 47,4 & 14 & 14 \\
\hline Ehe & 22,3 & 22,3 & 81,7 & 81,7 \\
\hline \multicolumn{5}{|l|}{ Beziehungsdauer } \\
\hline bis 4 Jahre & 35 & 35 & 7,9 & 7,9 \\
\hline 5 bis 6 Jahre & 30,1 & 30,1 & 14,8 & 14,8 \\
\hline 7 bis 9 & 21,4 & 21,4 & 19,8 & 19,8 \\
\hline 10 bis 14 Jahre & 9,3 & 9,3 & 27,6 & 27,6 \\
\hline 15 Jahre und länger & 4,2 & 4,2 & 30 & 30 \\
\hline \multicolumn{5}{|l|}{ Kinder } \\
\hline 1 Kind & & & 37,3 & 37,3 \\
\hline 2 Kinder & & & 45,4 & 45,4 \\
\hline 3 und mehr Kinder & & & 17,3 & 17,3 \\
\hline $\mathbf{N}$ & 986 & 986 & 1.807 & 1.807 \\
\hline
\end{tabular}

Quelle: pairfam 2008/2009, 1, Welle, eigene Berechnungen, ungewichtete Daten, 


\section{Tabellen}

Tabelle 1: Elternschaftsabsichten von Paaren in den nächsten zwei Jahren, kinderlose, Spaltenprozente

\begin{tabular}{lrr} 
& kinderlose Paare & Paare mit Kindern \\
beide Partner nein & $\%$ & $\%$ \\
beide Partner ja & 36,2 & 66,4 \\
Frau ja, Mann nein & 37,4 & 20,3 \\
Mann ja, Frau nein & 13,0 & 7,5 \\
& 13,3 & 5,8 \\
\hline
\end{tabular}

Quelle: pairfam 2008/2009, 1, Welle, eigene Berechnungen, ungewichtete Daten,

Tabelle 2: $\quad$ Einschätzung der Partnerschaftsqualität von Paaren, Spaltenprozente

\begin{tabular}{|c|c|c|}
\hline & $\begin{array}{c}\text { Paare } \\
\text { kinderlos }\end{array}$ & $\begin{array}{c}\text { Paare } \\
\text { mit Kindern }\end{array}$ \\
\hline & Kmotios & IIIL nimuern \\
\hline \multicolumn{3}{|l|}{ Beziehungszufriedenheit } \\
\hline beide weniger zufrieden bis unzufrieden & 1,8 & 3,5 \\
\hline beide zufrieden & 12,5 & 14,3 \\
\hline beide sehr zufrieden & 41,6 & 34,6 \\
\hline Mann zufriedener als Frau & 21,2 & 24,2 \\
\hline Frau zufriedener als Mann & 22,9 & 23,2 \\
\hline keine Angabe & 1,8 & 0,1 \\
\hline \multicolumn{3}{|l|}{ Trennungsabsicht } \\
\hline beide ja & 7,2 & 4,9 \\
\hline beide nein & 71,6 & 77,3 \\
\hline Mann ja/Frau nein & 5,3 & 3,6 \\
\hline Frau ja/Mann nein & 8,7 & 7,5 \\
\hline keine Angabe & 7,2 & 6,8 \\
\hline \multicolumn{3}{|l|}{ Ausmaß positiver Interaktion } \\
\hline beide niedrig & - & 0,7 \\
\hline beide mittel & 13,3 & 18,4 \\
\hline beide hoch & 44,3 & 26,4 \\
\hline Mann höher als Frau & 13,6 & 19,3 \\
\hline Frau höher als Mann & 18,7 & 22,1 \\
\hline keine Angabe & 10,1 & 13,1 \\
\hline \multicolumn{3}{|l|}{ Häufigkeit negatives Konfliktverhalten } \\
\hline beide selten bis nie & 31,1 & 26,3 \\
\hline beide manchmal & 16,9 & 12,9 \\
\hline beide häufig bis immer & 2,0 & 3,7 \\
\hline Mann häufiger als Frau & 14,1 & 17,4 \\
\hline Frau häufiger als Mann & 24,3 & 22,5 \\
\hline keine Angabe & 11,5 & 17,2 \\
\hline $\mathbf{N}$ & 986 & 1.807 \\
\hline
\end{tabular}

Quelle: pairfam 2008/2009, 1, Welle, eigene Berechnungen, ungewichtete Daten 
Tabelle 3: $\quad$ Ergebnisse der logistischen Regressionsanalyse, Determinanten des überinstimmenden Kinderwunsches von kinderlosen Paaren, Odds Ratios

\begin{tabular}{|c|c|c|c|c|c|c|c|c|}
\hline & \multicolumn{4}{|c|}{ Männer } & \multicolumn{4}{|c|}{ Frauen } \\
\hline & \multicolumn{4}{|c|}{ Odds Ratios } & \multicolumn{4}{|c|}{ Odds Ratios } \\
\hline & $3 a$ & $3 b$ & $3 c$ & $3 d$ & $3 e$ & $3 f$ & $3 g$ & $3 h$ \\
\hline \multicolumn{9}{|l|}{ Alter } \\
\hline unter 25 & 0,62 & 0,56 & 0,57 & 0,64 & $0,41^{*}$ & $0,42^{*}$ & $0,48+$ & $0,44+$ \\
\hline 25 bis 29 & $0,52^{\star *}$ & $0,56^{*}$ & $0,45^{* *}$ & $0,55^{\star}$ & 0,94 & 0,94 & 1,03 & 0,99 \\
\hline 30 bis $34(R G)$ & 1 & 1 & 1 & 1 & 1 & 1 & 1 & 1 \\
\hline 35 bis 39 & 0,82 & 0,86 & 0,74 & 0,83 & 0,59 & 0,6 & 0,66 & 0,66 \\
\hline 40 und älter & $0,44^{*}$ & $0,46+$ & $0,43^{*}$ & $0,37^{*}$ & $0,12+$ & $0,11+$ & $0,13+$ & $0,11+$ \\
\hline \multicolumn{9}{|l|}{ Herkunft } \\
\hline $\mathrm{BRD}(\mathrm{RG})$ & 1 & 1 & 1 & 1 & 1 & 1 & 1 & 1 \\
\hline DDR & $1,83^{\star *}$ & $1,83^{* *}$ & $1,75^{\star \star}$ & $1,79^{\star *}$ & $2,44^{\star \star \star}$ & $2,55^{\star \star \star}$ & $2,36^{\star \star \star}$ & $2,42^{\star \star *}$ \\
\hline Ausland & 1,51 & 1,54 & 1,68 & 1,38 & 0,99 & 0,9 & 0,92 & 1 \\
\hline \multicolumn{9}{|l|}{ Bildungsniveau } \\
\hline \multicolumn{9}{|l|}{ kein Abschluss/ } \\
\hline Hauptschule (RG) & 1 & 1 & 1 & 1 & 1 & 1 & 1 & 1 \\
\hline $\begin{array}{l}\text { Realschule } \\
\text { (Fach-)Hoch- }\end{array}$ & 1,34 & 1,34 & 1,45 & 1,34 & 0,84 & 0,86 & 0,85 & 0,85 \\
\hline schulreife/Abitur & 1,26 & 1,24 & 1,46 & 1,32 & 1,04 & 1,15 & 1,08 & 1,05 \\
\hline \multicolumn{9}{|l|}{ Erwerbsstatus } \\
\hline $\begin{array}{l}\text { nicht erwerbstätig } \\
\text { (RG) }\end{array}$ & 1 & 1 & 1 & 1 & 1 & 1 & 1 & 1 \\
\hline in Ausbildung & 0,82 & 0,86 & 0,76 & 0,78 & 0,54 & 0,56 & 0,57 & $0,45+$ \\
\hline teilzeitbeschäftigt & 2,01 & 1,77 & 1,63 & 2,05 & 0,74 & 0,8 & 0,8 & 0,68 \\
\hline voll erwerbstätig & 1,72 & 1,71 & 1,52 & 1,56 & 1,03 & 1,08 & 1,08 & 0,83 \\
\hline \multicolumn{9}{|l|}{ Religiosität } \\
\hline religiös & $1,51+$ & $1,54^{*}$ & $1,60^{*}$ & 1,42 & 1,25 & 1,3 & 1,25 & 1,37 \\
\hline nicht religiös (RG) & 1 & 1 & 1 & 1 & 1 & 1 & 1 & 1 \\
\hline \multicolumn{9}{|c|}{ Institutionalisierungsgrad } \\
\hline NEL ohne gem, $\mathrm{HH}$ & $\begin{array}{l}0,21^{* *} \\
0,46^{\star *}\end{array}$ & $\begin{array}{l}0,20^{* *} \\
0,46^{* *}\end{array}$ & $0,21^{* * *}$ & $0,19^{\star * \star}$ & $0,19^{\star \star \star}$ & $0,17^{\star * *}$ & $0,18^{\star \star \star}$ & $0,19^{* * *}$ \\
\hline NEL mit gem, $\mathrm{HH}$ & * & * & $0,50^{* * *}$ & $0,42^{* * *}$ & $0,36^{* * *}$ & $0,32^{* * *}$ & $0,37^{* * *}$ & $0,35^{\star \star *}$ \\
\hline Ehe $(R G)$ & 1 & 1 & 1 & 1 & 1 & 1 & 1 & 1 \\
\hline \multicolumn{9}{|c|}{ Beziehungszufriedenheit } \\
\hline $\begin{array}{l}\text { weniger zufrieden bis } \\
\text { sehr unzufrieden } \\
\text { zufrieden (RG) } \\
\text { sehr zufrieden }\end{array}$ & $\begin{array}{l}0,85 \\
1 \\
1,36+\end{array}$ & & & & $\begin{array}{l}0,72 \\
1 \\
1,34\end{array}$ & & & \\
\hline \multicolumn{9}{|l|}{ Trennungsabsicht } \\
\hline $\begin{array}{l}\text { ja } \\
\text { nein }(R G)\end{array}$ & & $\begin{array}{l}0,78 \\
1\end{array}$ & & & & $\begin{array}{l}1,23 \\
1\end{array}$ & & \\
\hline \multicolumn{9}{|c|}{ Ausmaß positiver Interaktion } \\
\hline $\begin{array}{l}\text { niedrig bis mittel (RG) } \\
\text { hoch }\end{array}$ & & & $\begin{array}{l}1 \\
0,81\end{array}$ & & & & $\begin{array}{l}1 \\
0,75\end{array}$ & \\
\hline \multicolumn{9}{|c|}{ Häufigkeit negatives Konfliktverhalten } \\
\hline $\begin{array}{l}\text { selten bis nie } \\
\text { manchmal bis häufig } \\
(R G)\end{array}$ & & & & $\begin{array}{l}0,97 \\
1\end{array}$ & & & & $\begin{array}{l}0,99 \\
1\end{array}$ \\
\hline & 783 & 773 & 755 & 750 & 778 & 758 & 750 & 748 \\
\hline
\end{tabular}

Quelle: pairfam 2008/2009, 1, Welle, eigene Berechnungen, ungewichtete Daten 
Tabelle 4: $\quad$ Ergebnisse der logistischen Regressionsanalyse, Determinanten des übereinstimmenden Kinderwunsches von Eltern, Odds Ratios

\begin{tabular}{|c|c|c|c|c|c|c|c|c|}
\hline & \multicolumn{4}{|c|}{ Männer } & \multicolumn{4}{|c|}{ Frauen } \\
\hline & \multicolumn{4}{|c|}{ Odds Ratios } & \multicolumn{4}{|c|}{ Odds Ratios } \\
\hline & $4 a$ & $4 b$ & $4 c$ & $4 d$ & $4 \mathrm{e}$ & $4 f$ & $4 g$ & $4 \mathrm{~h}$ \\
\hline \multicolumn{9}{|l|}{ Alter } \\
\hline unter 25 & 0.99 & 1.1 & 0.78 & 0.93 & $2,58^{*}$ & $2,53^{*}$ & $2,70^{*}$ & $2,71^{*}$ \\
\hline 25 bis 29 & 1.24 & 1.21 & 1.08 & 1.14 & $1,65^{\star}$ & $1,51 \mathrm{ç}$ & $1,59 c ̧$ & $1,59+$ \\
\hline 30 bis $34 R G$ ) & 1 & 1 & 1 & 1 & 1 & 1 & 1 & 1 \\
\hline 35 bis 39 & $0,67+$ & $0,67+$ & $0,61^{*}$ & $0,57^{*}$ & $0,60^{*}$ & $0,56^{*}$ & $0,56^{*}$ & $0,56^{*}$ \\
\hline 40 und älter & $0,36^{\star * *}$ & $0,35^{\star * *}$ & $0,34^{* * *}$ & $0,32^{\star * \star}$ & $0,16^{*}$ & $0,15^{*}$ & $0,19^{*}$ & $0,16^{*}$ \\
\hline \multicolumn{9}{|l|}{ Herkunft } \\
\hline $\mathrm{BRD}(\mathrm{RG})$ & 1 & 1 & 1 & 1 & 1 & 1 & 1 & 1 \\
\hline DDR & 1 & 1.01 & 1.03 & 1.01 & 0.87 & 0.89 & 0.85 & 0.93 \\
\hline Ausland & 1.41 & $1,45+$ & 1.34 & 1.4 & $1,42+$ & 1.37 & $1,44+$ & 1.4 \\
\hline \multicolumn{9}{|l|}{ Bildungsniveau } \\
\hline \multicolumn{9}{|l|}{ kein Abschluss/ } \\
\hline Hauptschule (RG) & 1 & 1 & 1 & 1 & 1 & 1 & 1 & 1 \\
\hline Realschule & 1.04 & 0.93 & 1 & 1 & 0.86 & 0.83 & 0.82 & 0.87 \\
\hline $\begin{array}{l}\text { (Fach-) Hoch- } \\
\text { schulreife/Abitur }\end{array}$ & $1,53^{*}$ & $1,44+$ & $1,48^{*}$ & $1,53^{*}$ & $1,44+$ & $1,47+$ & $1,44+$ & $1,49+$ \\
\hline \multicolumn{9}{|l|}{ Erwerbsstatus } \\
\hline nicht erwerbstätig (RG) & 1 & 1 & 1 & 1 & 1 & 1 & 1 & 1 \\
\hline in Ausbildung & 0.84 & 0.9 & 1.01 & 0.7 & 0.85 & 0.82 & 0.8 & 0.83 \\
\hline teilzeitbeschäftigt & 1.17 & 1.12 & 1.16 & 1.07 & 0.88 & 0.84 & 0.85 & 0.87 \\
\hline voll erwerbstätig & 1.07 & 1.04 & 1.02 & 1.03 & 1.09 & 1.01 & 1.04 & 1.05 \\
\hline \multicolumn{9}{|l|}{ Religiosität } \\
\hline religiös & $1,37+$ & 1.3 & $1,40+$ & 1.3 & $1,54^{\star *}$ & $1,43^{*}$ & $1,53^{* *}$ & $1,65^{\star \star}$ \\
\hline nicht religiös (RG) & 1 & 1 & 1 & 1 & 1 & 1 & 1 & 1 \\
\hline \multicolumn{9}{|c|}{ Institutionalisierungsgrad } \\
\hline NEL ohne gem. HH & 1.46 & 1.04 & 1.09 & 1 & 0.68 & 0.74 & 0.7 & 0.64 \\
\hline NEL mit gem. HH & 1.11 & 1.12 & 1.11 & 1.11 & 1.05 & 1.02 & 1.05 & 1.1 \\
\hline Ehe (RG) & 1 & 1 & 1 & 1 & 1 & 1 & 1 & 1 \\
\hline \multicolumn{9}{|l|}{ Kinder } \\
\hline 1 Kind (RG) & 1 & 1 & 1 & 1 & $\begin{array}{l}1 \\
0,17^{\star \star}\end{array}$ & $\begin{array}{l}1 \\
0,16^{* *}\end{array}$ & 1 & 1 \\
\hline 2 Kinder & $0,16^{\star * *}$ & $0,16^{\star \star *}$ & $0,17^{\star \star \star}$ & $0,17^{\star \star \star}$ & & & $0,18^{\star \star \star}$ & $0,17^{\star \star \star}$ \\
\hline 3 und mehr Kinder & $0,11^{\star \star \star}$ & $0,11^{\star \star *}$ & $0,10^{* * *}$ & $0,11^{* * *}$ & $0,10^{* *}$ & $\underset{*}{0,10^{* *}}$ & $0,09^{\star \star \star}$ & $0,09^{\star \star *}$ \\
\hline \multicolumn{9}{|c|}{ Beziehungszufriedenheit } \\
\hline $\begin{array}{l}\text { weniger zufrieden bis } \\
\text { sehr unzufrieden }\end{array}$ & $0,45^{\star *}$ & & & & 0.89 & & & \\
\hline zufrieden (RG) & 1 & & & & 1 & & & \\
\hline sehr zufrieden & 1.22 & & & & $1,60^{\star *}$ & & & \\
\hline \multicolumn{9}{|l|}{ Trennungsabsicht } \\
\hline ja & & $0,56^{*}$ & & & & $0,59^{*}$ & & \\
\hline nein $(R G)$ & & 1 & & & & 1 & & \\
\hline \multicolumn{9}{|c|}{ Ausmaß positiver Interaktion } \\
\hline niedrig bis mittel (RG) & & & 1 & & & & 1 & \\
\hline hoch & & & $0,70^{*}$ & & & & 0.79 & \\
\hline \multicolumn{9}{|c|}{ Häufigkeit negatives Konfliktverhalten } \\
\hline $\begin{array}{l}\text { selten bis nie } \\
\text { manchmal bis häufig }\end{array}$ & & & & $1,27+$ & & & & $1,56^{\star *}$ \\
\hline$(R G)$ & & & & 1 & & & & 1 \\
\hline $\mathbf{N}$ & 1,566 & 1,545 & 1,478 & 1,444 & 1,586 & 1,557 & 1,522 & 1,498 \\
\hline
\end{tabular}

Quelle: pairfam 2008/2009, 1, Welle, eigene Berechnungen, ungewichtete Daten 